\title{
The Distribution of the Informative Intensity of the Text in Terms of its Structure (On Materials of the English Texts in the Mining Sphere)
}

\author{
Ludmila Znikina ${ }^{1 *}$, and Elena Rozhneva ${ }^{1}$ \\ ${ }^{1}$ T.F. Gorbachev Kuzbass State Technical University, 650000, 28 Vesennyaya St., Kemerovo, Russia
}

\begin{abstract}
The article deals with the distribution of informative intensity of the English-language scientific text based on its structural features contributing to the process of formalization of the scientific text and the preservation of the adequacy of the text with derived semantic information in relation to the primary. Discourse analysis is built on specific compositional and meaningful examples of scientific texts taken from the mining field. It also analyzes the adequacy of the translation of foreign texts into another language, the relationships between elements of linguistic systems, the degree of a formal conformance, translation with the specific objectives and information needs of the recipient. Some key words and ideas are emphasized in the paragraphs of the English-language mining scientific texts. The article gives the characteristic features of the structure of paragraphs of technical text and examples of constructions in English scientific texts based on a mining theme with the aim to explain the possible ways of their adequate translation.
\end{abstract}

\section{Introduction}

In the research field of constructive effects of language discourse-analytical approaches are of interest as the ability of a structured and qualitative analysis of texts. The term "discourse" as it is known, originally began to be used in the sense of "functional style" as a particular type of texts: non-fiction, newspaper, scientific, technical etc., having their structural, lexical, and grammatical features [4]. Now we perceive this concept more widely, considering the discourse as a complex communicative phenomenon that includes the conditions of the actualization of texts. Naturally, these conditions include and extra-linguistic factors, such as the target installation of the sender. This makes them "pragmatic discourse" expressed appropriate communicative strategies in vocabulary, syntax, semantics, etc.

\section{Results and discussion}

We used discourse analysis to the study of English scientific texts of the coal and mining theme with the aim of identifying characteristics of the subsequent formalization of these

\footnotetext{
*Corresponding author: znikina@mail.ru
} 
texts. In other words, we consider discourse analysis as a practical receiving adequate transfer of the primary text into a derivative one.

The problem of unoriginality in the text formation and the actual texts, which are called derivatives, are the object of study of many researchers. This interest is quite natural, since there is too high proportion of derived texts in a text continuum in which we live.

As such we consider that a formal text in a summary essay, which is the result of clotting (and also the translation into the Russian language) of the English scientific and technical text. Issues of translation and the further formalization of foreign language scientific and technical texts categorized as theoretical and methodological, have always been a subject of interest, as in its complex, they relate to semantic aspects of language and lexical patterns of structural and systematic organization of the text. The study of these aspects provide, in our opinion, the availability and validity of necessary and sufficient perception of the adequacy of the information of a formal text.

In the theory of information systems semantic information is classified into primary and derivative semantic information [8], that is, the source of information, which is translated, may be called primary information. The relationships between elements of linguistic systems are structured and form a model of information transfer with a certain level of adequacy. To obtain a secondary semantic information - in this case the translation of scientific and technical texts from the English language and its formalization is a necessary lexical, grammatical, semantic and logical analysis of the source. From this point of view, getting derived semantic information is considered as a continuation of the process of cognition of the object and is a model of primary information. The degree of a formal conformance of this model with the source directly depends on the objectives of the translation, as well as the intentions and knowledge of the author (subjective factors). In other words, the relationship between the elements of the language system must be considered at two levels: the preservation of adequacy in the semantic coagulation, firstly, and, secondly, the adequacy of the translation.

Analysis of primary and derived texts is based on the assumptions of the constructive effects of language. So a translation of the abstract is the primary formalization of semantic information. Following to the theory of systems, the transformation scheme of the primary information into the derivative one (the transition from one language system (Language ${ }^{1}$ ) to another language system (Language ${ }^{2}$ ), it is possible to present a model for elements of these systems - the so-called constructive and neglecting attitude [4]. The first ratio shows the transition from the system with a simple language structure into a system with a more complex structure. Neglecting defines the reverse transition, which in our case corresponds to the formalized translation. Pragmatic factor, that is, a specific statement of the problem, determines the degree of formalization and, in this case, the sufficiency of the language system 2 (Language $^{2}$ ).

The translation of a foreign text into another language as a speech unit is complex from both a cognitive and linguistic point of view. And it's not just the lack of the necessary of the vocabulary or knowledge of grammar: when reading the text it is necessary to proceed not only from the lexical set but from the structure of the text as a whole, and from its structure's peculiarities[3]. A sign of linguistic organization is the source for the translation and formalization of scientific and technical texts: they are considered as a rule, from the point of view of availability of semantic and formal connections between text units (lexical units, sentences, with super-phrasal unities, paragraphs) as voice work in a multilevel and diverse aspect [2]. The absence of these links makes the text non-functional.

Naturally, when translating the foreign text becomes relevant to the issue of the adequacy of the semantic information of the language system. In our view, the translation with the specific objectives and information needs of the recipient must be understood under the appropriate translation of scientific and technical text but not its exhaustive accuracy. If the translation is considered to be adequate and it provides the coincidence of the denotative 
structure and forms of representationof the information, then the attitude to the language coagulation of this information (a summary, an abstract) suggests, obviously, a change of not denotative unity of the text, but the frame structure of the utterance. So, the translation is carried out by means of grammatical and structural changes. Such goal setting is also an adequate translation. However, it should not be considered pragmatically appropriate, because in this case it is based on a change of the communication setup, inherent in the source.

In any case, the correct solution of the problem of derived semantic information (Language $^{2}$ ), is adequate to the primary one (Language ${ }^{1}$ ). Otherwise, this would contradict the structural, analytical and logical transformation of information. The essence of such conversions is not in receipt of the formal copy, is is in preserving the communicative significance.

In this case we are dealing with a super-phrasal unities. Such units include the paragraph, graphically, placing the text and at the same time separating it into information segments. The structure of a paragraph, the scientific and technical text is not very diverse, due to which they can model. The technical text is characterized by the presence of key sentence in a paragraph. In a concentrated form, the key sentence conveys the information contained in the paragraph, and can be in any part of it [6]. However, the vast majority of the paragraphs it is in the initial position and serves as a subtitle of the paragraph ( a method of introductory or screening reading of a text is based on it: paragraph by paragraph information search requires the ability to extract the basic elements of the meaning from the original quickly enough) [7].

Informative important segments occupy a certain logical position in the structure of the text.

We consider (here and later) some examples of texts taken from the mining sphere [1]. In the scientific and technical article these segments occupy the vast majority of the starting position: the first paragraph and the first sentences of the middle paragraphs of the text. From the boundary position of the whole text, the priority in terms of informativeness is given to the first paragraph.

For example:

1. The methane content in coal bed can be estimated: a) directly in situ by desorption of the coal steam; b) under laboratory conditions by determination of the gas amount incorporated into the extracted coal.

2. The collected data made it possible to prepare models for the sedimentary environment of the coal beds on two different levels:

- General level, prepared from the study of inorganic/organic macrofacies relations, using mostly results of field studies and borehole lithological analysis;

- $\quad$ Detailed level, prepared from the study of microbotanical and micropetrographic features of coal.

3. As generally known, the problem of prevention for coal self-heating and self-ignition is of great importance. Such scientists as E. Pilarczyk, P. Leonhardt, W. Wanzl, D. Rusezev, K. Markova, N. Oils, X. Dong, D. Drysdale, A. C. Smith, P. Panek, B. Taraba, E. Sebestova and others study the process of coal oxidation and different inhibitors for suppressing of coal self-ignition.

4. In contrast, the outlook for Indonesian coal miners is more positive. Two key trends will develop during 2017 and beyond. Firstly, an increase in production that will outperform expectations as Indonesia seeks to capitalise on growing demand for coal from not only China but increasingly other Asian emerging markets, such as Vietnam, Malaysia and the Pilippines. Secondly, there will be a sustained rise in domestic demand that will underpin higher production levels as the government invests heavily in power infrastructure and ramps upcoal as a share of Indonesia's energy mix.

5. Coal demand, and in particular thermal coal deman,hasincreased significantly from 2012 after Japan'snuclear reactors were switched off following the Tohoku Earthquake in 2011. 
This are the first paragraphs of scientific-technical articles. The accuracy requirement led to the emergence of the syntactic feature such as information intensity of the sentences. The need to express a complex system of scientific concepts, to establish relationships between them creates a complex and complicated sentences, which can hold much information. These segments are informative significant, as they clearly state the key ideas of the article provided, for example, using modal structure can be estimated. This clarity is achieved by dividing key ideas into paragraphs in the first and second examples. The emphasis on key words is done graphically through the colon. In addition, the third, forth and fifth examples introduce the phrases As generally known (as you know), in contrast, in particular which make the reader pay special attention to it, as different causal relationships and the relationships as moments of universal interconnection, "communications", "chain of causes" are chiefly important in scientific discourse. It is impossible to execute and perceive complex logical and conceptual relationships of the content of scientific texts without this, as well as strict, accented logical coherence of scientific discourse is aslo not possible [5].

The structural and systematic organization of the scientific and technical text is quite clear: in most cases it consists of thesis, arguments and conclusion with the presence of the constituents to argue, to convince, to raise a scientific interest.

In the distribution of information intensity by means of pragmatic impact to enhance persuasion and argumentation such units as: in addition to, for example, for instance, etc, and so on are frequently used. Modal and cognitive verbs, including a component of "knowledge" (to convince, to persuade, etc.) strengthen the structure.

Let's consider some examples on this piece of information:

1. In addition, while there is a current oversupply domestically, India's coal usage is set to continue to rise as the government seeks to increase power capacity.

2. For example, coal from South Kalimantan mines can arrive in Guangzhou after 7-8 days, while Chinese mined coal from large mines at Shanxi can take a total of 8-9 days to arrive in Guangzhou.

Since the main function of this type of the text is a communicative and evaluative function, the basic modality of its structure always remains the subject (objective) modality.

An example of the structure from an English scientific and technical text representing not only the modalities but also the position of the modal structure is the following sentence:

Therefore we must confine ourselves to a brief survey.

The use of the modal verb "must", in addition to the introductory word "therefore", is taken for greater emphasis of the reader's attention on the key ideas of the article.

Let's consider another example:

The effect of temperature under 1MPa partial pressure of oxygen for $30 \mathrm{~min}$ on the solid product yield of the lignite from Gediz area is shown in Figure 2.

This is the first sentence of the second paragraph, which is the focus of an expandable informative article: there are accurate data and a link to the picture.

Thus, it is in the first position of the text, especially in the first paragraph, we observe a saturation of informative sentences.

An approach of search and retrieval of information in the process of discourse analysis of text paragraph by paragraph is key when you minimize or formalize it, including when the information is any text a summary, an abstract, a reference, a review, etc.). If we continue our discussion specifically about the translation of a foreign-language text, the logical partitioning of a paragraph (definition of rheme and theme) suggests the formalization of the text with a sufficient level of adequacy of translation. The term "content" is a set of objects and their characteristics. Under the certain restriction on the lexical and syntactic levels in the process of formalizing the necessary condition for the correct transmission of the content of the text is to identify the key positions in the paragraph, the choice of the adequate level of 
nuclear structures to the original and correct (relevant from the point of view of pragmatics and language norms) of the translated equivalent.

\section{Conclusion}

Using elements of a discourse analysis of a scientific text, we investigate compositional and content aspects of the process of formalization of the text from the following viewpoint: studying those segments which are rich in information in correlation with the structural and graphic design of the text (paragraph by paragraph); identifying of logical and conceptual relations expressed by lexical features of the discourse, which were essential to the perception and design of the logical coherence of scientific statements and, accordingly, the subsequent adequacy of the text with derived semantic information.

\section{References}

1. D. Geeraerts, Theories of lexical semantics, 336 (Oxford University Press Inc., New York, 2010)

2. V. A. Kolosova, Modern problems of science and education, 6, 567 (2013)

3. J. Peregrin, V. Svoboda, Synthese, 14:190, 2897 (2013)

4. S. Pohorec, M. Verlič, M. Zorman, Proc. of the 14th east European conference on Advances in databases and information systems (ADBIS'10), 445-453 (2010)

5. P.H. Portner, What is Meaning? Fundamentals of Formal Semantics (Oxford, Blackwell, 2005)

6. N.M. Solomatin, Information Semantic Systems (High School, 1989)

7. L.S. Znikina, N. A. Zaostrovskaya, World of science, culture, education, 4:2,142 (2011) 
